\title{
Bacteriological Study and Pathological Features of Necrotic Dermatitis in Broilers in Eastern Algeria
}

\author{
Mourad ZEGHDOUDI ${ }^{1 *}$, Latifa MERDACI ${ }^{1}$, Seloua MORSLI ${ }^{1}$, Mardja TAHRI ${ }^{2}$, Leila AOUN ${ }^{2}$ \\ ${ }^{1}$ Department of Veterinary Sciences, Chadli Benjedid University, El-Tarf-36000, Algeria \\ ${ }^{2}$ Department of Biological Sciences, Chadli Benjedid University, El-Tarf-36000, Algeria \\ *corresponding author: mouradzeg@yahoo.fr
}

Bulletin UASVM Veterinary Medicine 77(1)/2020

Print ISSN 1843-5270; Electronic ISSN 1843-5378

doi:10.15835/buasvmcn-vm: 2019.0017

\begin{abstract}
The various descriptions reported of necrotic dermatitis and its pathogenesis remains ambiguous. The purpose of this study is to suggest some pathognomonic characters of the disease with effective preventive and curative measures. Dead birds presented sudden death, greenish lesions at the wings and abdomen with adjacent tissues destruction. The distribution of lesions on 103 dead birds at 45th day of age showed that the right wing and the right abdomen are the most affected. The disease caused a mortality average rate of $12,8 \%$ in a period of 5 days and treatments based on several antibiotics showed that only ampicillin and oxytetracycline gave positive results. The use of API 20 A kit confirmed the presence of Clostridium septicum. The present study showed that necrotic dermatitis must be differenced from others skin affections. Qualities of litter, pathological diagnosis, rapid and judicious treatment, are the most effective means to control this disease.
\end{abstract}

Keywords: ampicillin, broiler, Clostridium septicum, Elisa, greenish wing

\section{Introduction}

Clostridia are ubiquitous in both environment and intestinal tract of healthy animals. Clostridial diseases in birds were often reported as cholangiohepatitis, ulcerative enteritis and necrotic enteritis, however necrotic dermatitis remain the less investigated. It is a disease of chickens and turkeys that causes severe economic losses in the poultry industry worldwide and the main gateway of the microorganisms is done by skin lesions (Gornatti-Churria et al., 2018). In the US, this disease in commercial broiler operations is in increase (Tabler and Wells, 2016).

Clostridial dermatitis was known such as cellulitis, malignantedema, gangrenous dermatitis, blue wing disease and caused by Clostridium perfringens, Clostridium sordelli, Escherichia coli,
Streptococcus sp. and Staphylococcus aureus (Mandal and Rath, 2015). The disease continues to cause severe losses due to its late onset in adult (Norton et al., 2000). The recent studies reported that the pathogenesis of necrotic dermatitis is not known, there is no consensus on how the disease is spread. The control of the disease by using different antibiotics remained insufficient and new studies include supplementation of yeast in feed to combat necrotic dermatitis (Huff et al., 2014).

Being that this disease is confounded with other skin diseases, we suggest to present a detailed description of the disease different to those reported previously and to propose a judicious treatment. 


\section{Materials and methods}

The study was performed during the winter 2018 in five broiler's farms located in the north east of Algeria. All the flocks were reared on a litter of straw from the same origin. Average rate of mortality was $5.2 \%$ until the age of 36 days. Pathological troubles occurred from the 37 th day caused a rapid raise of mortality which has reached peaks of above a hundred dead birds per-day with an average mortality rate of $12.8 \%$ in a period of 5 days. The symptoms were observed daily and necropsy on dead birds was performed every day on site. Different antibiotics such as enrofloxacin, amoxicillin, erythromycin, ampicillin, oxytetracycline and sulfamids were administrated to control the disease. The antibiotics administration was done with the available products at the beginning of the disease without using the antimicrobial susceptibility testing. Dead birds were brought under aseptic conditions to laboratory of epidemiology research of Chadli Bendjedid University (ESSPRETCADS) and samples of subcutaneous tissues from the abdomen and wings were placed in cultures.

Identification of Clostridium was performed on the basis of growth characteristics on agar containing blood after anaerobic incubation at 35$37^{\circ} \mathrm{C}$ for $18-24$ hours. The results of Gram stain

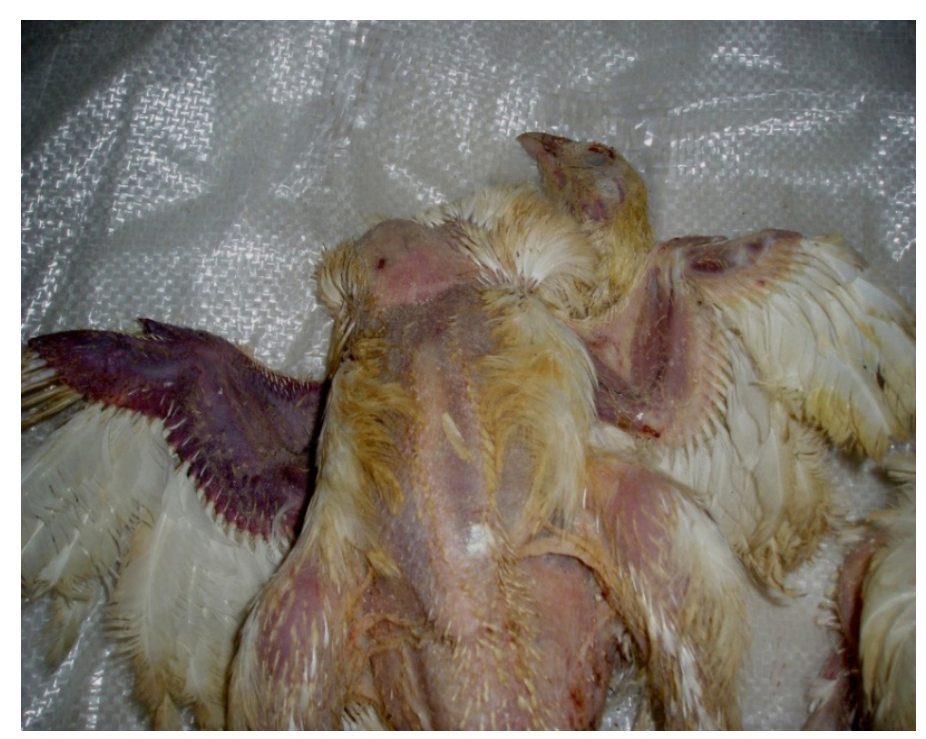

Figure 1. Necrotic dermatitis lesions from the broiler's right wing aged of 37 days

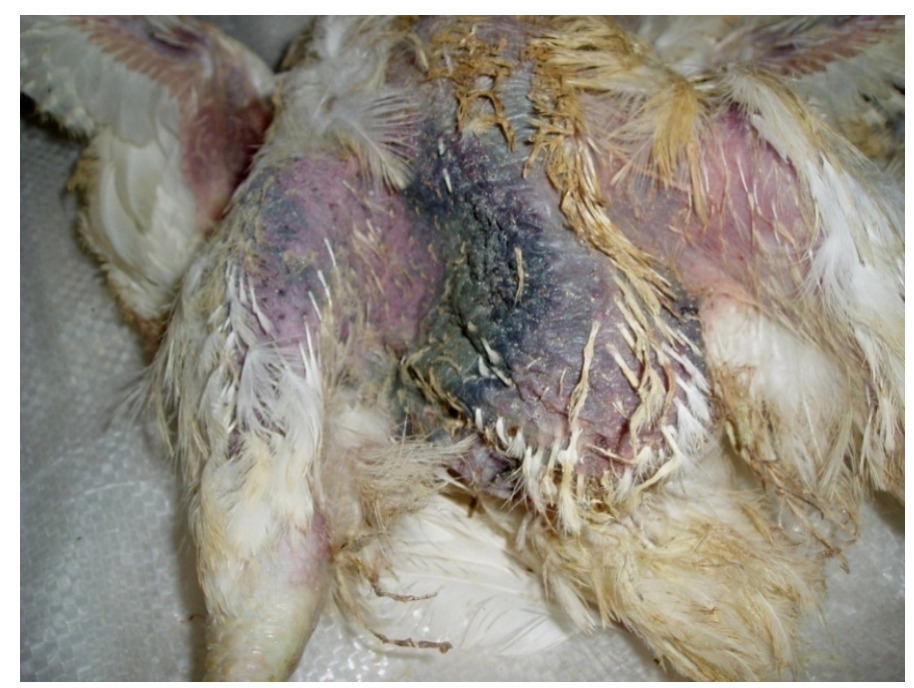

Figure 2. Necrotic dermatitis lesions from the broiler's right part of abdomen aged of 37 days 
and microscopic morphology of colonies were used to confirm the identification by using the API 20 A (Biomerieux, France) system which enables 21 tests to be carried out for the biochemical identification of anaerobes. The reactions are read according to manufacturer's instructions and the identification was obtained by referring to the analytical profile index.

\section{Results and discussions}

In the present study, we recorded that all the dead birds had greenish lesions exclusively at the wings (Figure 1) and abdomen (Figure 2) with putrefaction of adjacent tissues.

Distribution of lesions on 102 dead birds showed that the lesions were mostly located on the right wing and the right abdomen of birds (Table 1). This distribution of lesions attested that the wings and the side parts of abdomen were the most exposed to litter which represented the source of contamination. We conclude that birds have preference for the right lateral recumbency and the route of infection must be through the healthy integument by the feathers bulbs.

Some theories suggested that the clostridia contaminate the environment and then penetrate the bird's circulation by the oral infection and the damaged intestinal wall. Another theory suggests that clostridia enter directly through abraded skin where caused inflammation gas formation under the skin (Umar et al., 2015). Char (1986) cited that the gross lesions are black on a wet area of the skin, devoid of feathers at the wings, chest, abdomen or legs. The areas of the skin most affected are the abdomen, head, thigh and wing (Tabler and Wells, 2016).

Ritter (2008) reported that the presumed route of infection must have been through the damaged integument because dermatitis lesions are found close to interrupted areas of the skin. In the present study colonies on blood agar plates were 1 to 4 $\mathrm{mm}$ of diameter, circular, gray, translucent with irregular margins. Bacterioscopically the spores are oval and sub terminal. Biochemical tests using Api 20 A have demonstrated that Clostridium septicum is the main agent responsible for necrotic dermatitis. Shirasaka and Benno (1982) reached to the same result whereas Swayne (2017) cited that necrotic dermatitis is caused by one of three agents: $C$. perfingens types A and C, $C$. septicum or Staphylococcus aureus. Neumann et al. (2006) reported that $23.5 \%$ of disease lesions were positive for $C$. perfringens, $41.2 \%$ for $C$. septicum and $29.4 \%$ for both species. Escherichia coli and Streptococcus spp. have occasionally been isolated from birds diagnosed with cellulitis (Kahn, 2010). This disease with important loses suggests to realize the identification of the bacterial species by the use of the PCR as a perspective during the next works. Treatments based on ampicillin and oxytetracycline reduced the mortality at the second and the third day of treatment respectively and a total recovering of animals at the $5^{\text {th }}$ day (Tab. 2 ). No others antibiotics or sulfamids used in this study have given any positive results. Additional, a nebulization by using chlorinated water was applied on the litter.

Some reports of treating litter with alum, aluminum sulfate, sodium sulfate, salt reduced the incidence of necrotic dermatitis (Clark et al., 2010). Use of probiotics, acidifiers, vitamins and windrowing technologies can reduce the onset of necrotic dermatitis by reducing high levels of Clostridium (Clarketal., 2004). Necrotic dermatitis can be treated by copper sulfate in water (Waneck, 2010), Penicillin and Erythromycin (Fowler and Hussaini, 1975). The present study showed that occurring of necrotic dermatitis in a wet region during the winter and the use of the same litter represented the main factors which have favored the contamination of the broiler flocks. Similarly, Ritter (2008) revealed that higher

Table 1. Localization of necrotic dermatitis lesions on 102 dead birds

\begin{tabular}{ccccc}
\hline $\begin{array}{c}\text { Affected } \\
\text { Body's part }\end{array}$ & Right wing & $\begin{array}{c}\text { Right } \\
\text { abdomen }\end{array}$ & $\begin{array}{c}\text { Right wing with } \\
\text { right abdomen }\end{array}$ & $\begin{array}{c}\text { Left wing with } \\
\text { right wing }\end{array}$ \\
\hline Number of birds & 81 & 07 & 12 & 33 \\
\hline Rate \% & 78.64 & 6.79 & 11.65 & 2.91 \\
\hline
\end{tabular}

Note: Lesions are located to more than $90 \%$ of cases at the right parts of birds. 
Table 2. Results of administrated treatments

\begin{tabular}{lccccc}
\hline \multicolumn{1}{c}{ Flocks } & $\mathbf{1}$ & $\mathbf{2}$ & $\mathbf{3}$ & $\mathbf{4}$ & $\mathbf{5}$ \\
\hline Number of birds & 4000 & 5000 & 5500 & 6500 & 6000 \\
\hline Age of disease occurrence & 36 days & 37 days & 36 days & 36 days & 38 days \\
\hline $\begin{array}{l}\text { Treatments } \\
\text { Enrofloxacyne }\end{array}$ & Erythromycin & Sulfamids & Ampicilline & Oxytetracycline \\
\hline $\begin{array}{l}\text { Mortality during } \mathbf{5} \\
\text { days of treatment }\end{array}$ & $10.5 \%$ & $12.5 \%$ & $11.7 \%$ & $3.1 \%$ & $5.3 \%$ \\
\hline
\end{tabular}

moisture percentage in litter and high humidity in environment predisposes broiler flock to necrotic dermatitis. However, Willouhby (1996) reported that the peak was observed during the summer, a poor quality straw and bad management in the distribution of water. Okonkwo and Madubuike (2015) reported an epidemic of gangrenous dermatitis in broiler chickens reared for 4 weeks in deep litter before being transferred to battery cages; however, lesions are not characteristic. It has been observed in the US that litter clearing delays the recurrence of necrotic dermatitis epidemics in poultry houses from 2 to 3 growth cycles, but does not eliminate the problem (Rommel et al., 2013). In this study, average rate of mortality was $5.2 \%$ until the age of 36 days. Pathological troubles occurred from the $37^{\text {th }}$ day caused a rapid mortality rate peaked at more than 100 birds/day with an average mortality rate of $12.8 \%$ in 5 days. Those finding were similar to those reported by Fowler and Hussaini (1975). Mandal and Rath (2015) observed that Clostridial dermatitis usually strikes adults. The severity of diseases is found to be more during 17 to 20 weeks age group. Necrotic dermatitis is marked by a sudden increase in mortality occurring from 5 to 7 weeks and from 12 to 20 weeks of age in chicken and turkeys respectively (Ritter, 2008). Clinical signs observed are represented by ataxia, depression and sudden death. Shivaprasad (2016) observed depression, lack of coordination, lateral recumbency, leg weakness, ataxia but a total mortality of $2.0 \%$ during an epidemic period of 5 days.

\section{Conclusion}

This study showed that necrotic dermatitis is caused by Clostridium septicum. The clinical signs and lesions are pathognomonic and different from the others skin diseases while litter is considered as the main source of contamination. Exclusive location of necrotic dermatitis lesions on the right wing and right side of abdomen demonstrated that birds preferred the right lateral recumbency and contamination was realized through the healthy tegument. Quality of the litter and quick treatment with ampicillin are the best way to control this disease.

Acknowledgements. This study was fully supported by Laboratory of Epidemiology Research of Chadli Bendjedid University, El Tarf, Algeria.

\section{References}

1. Char NL (1986). A rare occurrence of clostridial infection in poultry. Poult Advis 19: 59- 62.

2. Clark AG, Porter P, Brian B, Mc Comb C, Lipper R, Olson DS, Sheilina E, Nohner E, Shivaprasad F (2010). Clostridial dermatitis and cellulitis: an emerging disease of turkeys. Avi Dis 54: 788-794.

3. Clark D, Watkins S, Jones F, Norton B (2004).Understanding andcontrol of gangrenous dermatitis in poultry, United Poultry Concerns, Inc. PO Box 150 Machipongo, VA 23405-0150.

4. Fowler NG, Hussaini SN (1975). Clostridium septicum infection and antibiotic treatment in broiler chickens.Vet Rec 96:14-15.

5. Gornatti-Churria CD, Crispo M, Shivaprasad HL, Uzal FA (2018). Gangrenous dermatitis in chickens and turkeys. J Vet Diag Invest 30 (2):188-196.

6. Huff GR, Huff WE, Ratha NC (2014). Effects of vitamin D and yeast extract supplementation on turkey mortality and clostridial dermatitis incidence in a dexamethasone immune suppression model. Avi Dis 58:572-578.

7. Kahn CM (2010). Gangrenous dermatitis. In the Merck Veterinary Manual, 10th Ed. S. Line Pub.Merck \& Co., Inc., Whitehouse Station, New Jersey.

8. Mandal KD, Rath PK (2015). Clostridial dermatitis: A New Concern among Turkey Farmers. Res J Recent Sci 4:1-6 
9. Neumann T, Ritter D, Dunham S, Skalecki J, Rehberger G (2006). Characterization of Clostridium from broiler farms experiencing recurrent outbreaks of gangrenous dermatitis. Poultry science association, annual meeting Edmonton, Alberta, Canada.

10. Norton RA, Hess JB, Bilgili SF (2000). Gangrenous dermatitis reemerges in broilers. WATT Poult 1: 38-39.

11. Okonkwo C, Madubuike K (2015). An outbreak of gangrenous dermatitis in broilerchickens reared on battery cage operation in Umuahia, Abia State Nigeria. J Vet Advis 5: 819-825.

12. Ritter GD (2008). Pathogenesis of gangrenous dermatitis in chickens and attempts at experimental reproduction. In: Proceedings Midwest Poultry Federation Convention, St. Paul, Minneapolis.

13. Rommel M, Tan TSL, Bautista DA, Phillips K, Tablante NL (2013). Descriptive study of microbial profile of poultry litter from broiler farms with and without a history of gangrenous dermatitis and litter from an experimental poultry house. J Appli Poult Res 22(2): 344-350.
14. Shirasaka S, Benno Y (1982). Isolation of Clostridium septicum from diseased chickens in broiler farms. Japan J Vet Sc 44: 807-809.

15. Shivaprasad HL (2016). Gangrenous dermatitis in poultry. In: Uzal FA, eds. Clostridial diseases of animals, Ames IA: Wiley-Blackwell.

16. Swayne DE (2017). Gangrenous dermatitis In : diseases of poultry $13^{\text {th }}$ ed. Chapter 22 .

17. Tabler GT, Wells BJ (2016). Gangrenous Dermatitis in Broilers, Mississipi State University.

18. Umar S, Nawaz S, Shahzad M, Munir MT, Shah MAA (2015). Emerging issue of gangrenous dermatitis in broilers. J Avi Res 1(2):17-19.

19. WaneckCR(2010). Preventivemeasuresto controlclostridial outbreaks of gangrenous dermatitis in commercial broiler operations. MS Thesis. Texas A\&M University.

20. Willouhby DH (1996). Periodic recurrence of gangrenous dermatitis associated with Clostridium septicum in a broiler chicken operation. J Vet Diag Invest 8: 259-261. 